\title{
Investigation of the Magellanic eclipsing binary HV 2241
}

\author{
P. G. Ostrov ${ }^{1, \star}$, N. I. Morrell ${ }^{1,3}$, and E. Lapasset ${ }^{2,3}$ \\ 1 Facultad de Ciencias Astronómicas y Geofísicas, Universidad Nacional de La Plata, Paseo del Bosque S/N, \\ 1900 La Plata, Argentina \\ e-mail: nidia@fcaglp.edu.ar \\ 2 Observatorio Astronómico, Universidad Nacional de Córdoba, Laprida 854, 5000 Córdoba, Argentina \\ e-mail: lapasset@mail.oac.uncor.edu \\ 3 Member of the Carrera del Investigador Científico, CONICET, Argentina
}

Received 25 April 2001 / Accepted 14 August 2001

\begin{abstract}
We present a CCD $V$ light curve of HV 2241, together with some observations in the $B$ band. We analysed our photometric data jointly with previously published radial velocities by means of the Wilson-Devinney code, and derived new values for the physical parameters of this massive eclipsing binary system. We confirm that this system is semi-detached, with the secondary (less massive and less luminous component) filling its Roche-lobe. From our analysis, we estimate the following masses and radii for the components of HV 2241: $M_{1}=36.2 \pm 0.7 M_{\odot}$, $R_{1}=14.9 \pm 0.4 R_{\odot}, M_{2}=18.4 \pm 0.7 M_{\odot}$ and $R_{2}=13.7 \pm 0.4 R_{\odot}$. We review the spectral classification analysing HST and IUE data and discuss the temperature and the reddening for this star.
\end{abstract}

Key words. binaries: eclipsing - stars: early-type - stars: fundamental parameters - stars: individual: HV 2241

\section{Introduction}

The study of double-lined eclipsing binaries provides the fundamental data needed for the elaboration of physical models of stars. Massive systems are of particular interest, since massive star models present some challenging difficulties: Stellar evolutive models for the hottest stars require masses of the order of $\sim 100 M_{\odot}$ (see, for example, Schaerer et al. 1993 for models for stars with LMC metallicity), while the highest binary stars masses empirically determined, i.e. the highest Keplerian masses, hardly surpass $\sim 50 M_{\odot}$ (Schweickhardt et al. 1999; Antokhina et al. 2000; Ostrov 2001). Only a handful of systems containing massive stars have reliable parameter estimates (see, for example, Burkholder et al. 1997). The modelling of close binaries that evolve via mass exchange are particularly problematic, since it is not clear how much material the system losses during mass transfer (Vanbeveren et al. 1998; Wellstein et al. 2001). Observations of systems of different masses at several stages of evolution are needed in order to obtain constraints that aid numerical evolutive

\footnotetext{
Send offprint requests to: P. G. Ostrov, e-mail: ostrov@fcaglp.edu.ar

* Visiting Astronomer, Complejo Astronómico El Leoncito operated under agreement between the Consejo Nacional de Investigaciones Científicas y Técnicas de la República Argentina and the National Universities of La Plata, Córdoba and San Juan.
}

modelling to address those problems. The acquisition of data from Magellanic Cloud stars has an additional interest since it allows one to explore the evolutive scenario in a low metallicity environment. For these reasons, we have started a campaign to obtain accurate photometry of some interesting massive systems in the Magellanic Clouds.

The Harvard variable HV 2241 was one of the objects included in our program since the beginning, because this star had a published radial velocity curve (Niemelä \& Bassino 1994) and was a known to be an eclipsing binary, with a photographic light curve available from Gaposhkin (1970), who determined a period of 4.342634 for this system. An analysis of that light curve was performed by Russell (1956). A fragmentary photoelectric $U B V$ light curve is available from Davidge (1987), who also presented an analysis of his data. Niemelä \& Bassino (1994) determined a radial velocity curve for this system. Based on their spectroscopic observations, they classified the binary components as $\mathrm{O} 7 \mathrm{~V}+\mathrm{O} 8 \mathrm{~V}$-III, and estimated the fundamental parameters of the system. More recently, Pritchard et al. (1998) (hereafter PTCG) obtained CCD photometry in the Strömgren ubvy and Cousins $I$ filters, their light curves being particularly complete in the $u$ and $I_{\mathrm{C}}$ bands. They analysed their data together with the radial velocities from Niemelä \& Bassino (1994) obtaining a solution for the system. Based on their multi-band photometry, PTCG also estimated a reddening for this star and derived surface temperatures, obtaining values which are 
Table 1. Coordinates, photometric data and spectral types for HV 2241.

\begin{tabular}{lll}
\hline$\alpha$ & $4^{\mathrm{h}} 57^{\mathrm{m}} 15^{\mathrm{s}} 8$ & $\mathrm{~J} 2000$ \\
$\delta$ & $-66^{\circ} 33^{\prime} 56^{\prime \prime}$ & $\mathrm{J} 2000$ \\
$l$ & 277.1360 & \\
$b$ & -36.0268 & \\
$V$ & $13.510 \pm 0.013^{\mathrm{a}}$ & from PTCG \\
& $13.52 \pm 0.015^{\mathrm{b}}$ & this work \\
& $13.47 \pm 0.015^{\mathrm{c}}$ & $"$ \\
$B-V$ & $-0.039 \pm 0.015^{\mathrm{c}}$ & $"$ \\
$E(B-V)$ & $0.174 \pm 0.020^{\mathrm{a}}$ & PTCG \\
& $0.270 \pm 0.020^{\mathrm{b}}$ & this work \\
Sp. types & O7 V+O8 V-III & Niemelä \& Bassino (1994) \\
& O7 V+B0 III & Niemelä \& Morrell (1999) \\
& O7 III+B0 III & this work \\
\hline
\end{tabular}

\footnotetext{
a For $\phi=0.832$;

b Converted to $\phi=0.832$;

c Measured at $\phi=0.201$.
}

remarkably low in comparison with those expected from the published spectral types.

In this paper we present a new CCD $V$ light curve for HV 2241, together with some observed points in the Johnson $B$ band. We analyse this data together with the radial velocity determinations from Niemelä \& Bassino (1994), deriving new values for the fundamental parameters of the system. Also, we review the spectral classification analysing HST and IUE data and we obtain a new estimation for the reddening for this star. In Table 1 we present a summary of the coordinates, photometric data and spectral types derived for HV 2241.

The paper is organized as follows: Sect. 2 describes the observations and reduction procedures; Sect. 3 describes the analysis of the data; Sect. 4 discusses the results and Sect. 5 presents our conclusions.

\section{Observations and reduction procedures}

The CCD observations analysed in this paper were acquired with the 2.15-m telescope at CASLEO (San Juan, Argentina), during three runs in 1995, 1997 and 1998. Table 2 gives a summary of each observing run and Fig. 1 displays the region around HV 2241 taken from one of our CCD V frames.

Aperture photometry was performed by means of the DAOPHOT II program (Stetson 1987, 1991) using an aperture of $4.75^{\prime \prime}$ radius. For differential photometry, some 18 field stars were selected as local standards, and were used to transform all the measurements to a unique instrumental system. The fully detailed description of this procedure was presented in the first paper of this series, Ostrov et al. (2000).

Transformation equations to the standard system were derived through observations of the Selected Areas 92 and 98 of Landolt (1992). Although in Ostrov et al. (2000)

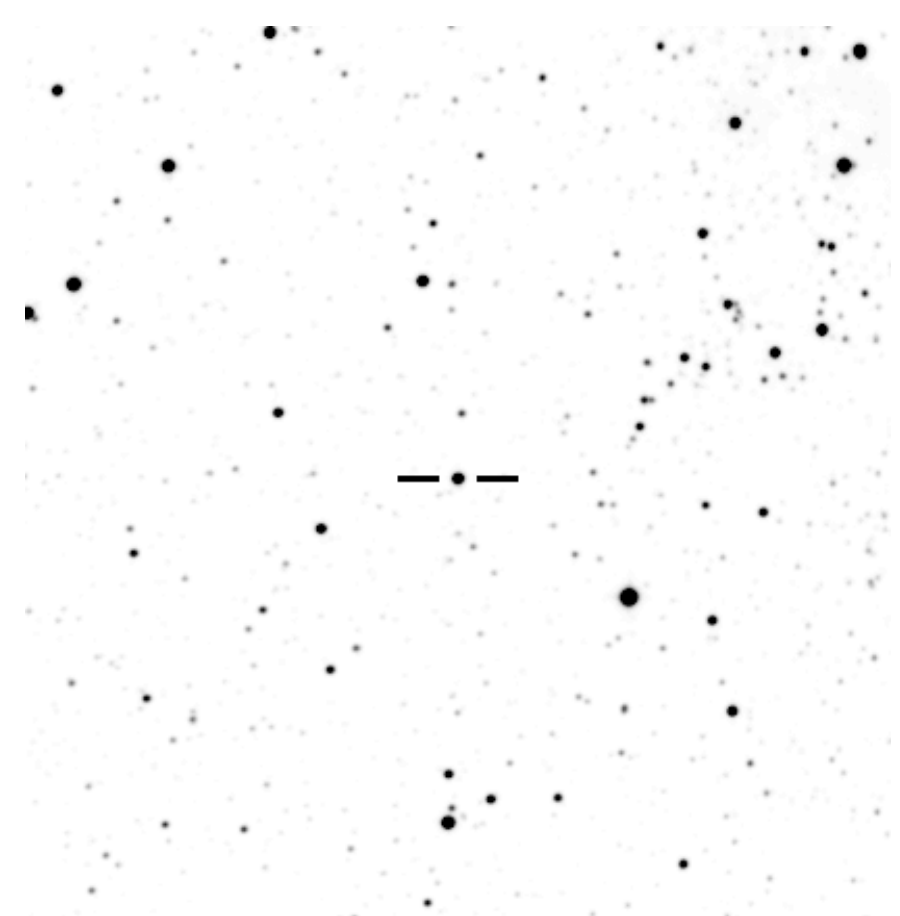

Fig. 1. Finding chart for HV 2241. North is at the top and East is at the left. The size of the region displayed is approximately $6^{\prime} \times 6^{\prime}$

we used mean extinction coefficients from Minniti et al. (1989), we now attempted to derive our own extinction coefficients, although we do not have standards observed with $X>1.5$. Our own estimates for the first order extinction coefficients were $K_{V}=0.19 \pm 0.02$ and $K_{B-V}=$ $0.087 \pm 0.02$, not significantly different from the published ones $\left(K_{V}=0.165 \pm 0.011\right.$ and $\left.K_{B-V}=0.11 \pm 0.01\right)$.

We used $B$ and $V$ images of the HV 2241 field acquired at HJD $=2450772.715(\phi=0.201)$ to tie our instrumental system to the standard system. The measured magnitude and colour for this phase were $V=13.47 \pm 0.015$ and $(B-V)=-0.04 \pm 0.015$.

Tables 3 and 4 display the final standard magnitudes derived for HV 2241, together with the internal rms residuals derived from the local calibrators, the airmass and the $F W H M$ for each frame.

\section{Data analysis}

\subsection{Ephemeris}

We observed a primary minimum during the 1997 run and two secondary minima during 1997 and 1998, the secondary minimum of 1997 being the best covered one. Assuming that the orbit is circular, we estimated a new time of primary minimum, $E_{0}=2450771.840 \pm$ 0.002. Combining this time of minimum with the data from Payne-Gaposchkin (1971), we derived a period of $4.342635 \pm 0.0000015$, that is virtually identical to the one given by Payne-Gaposchkin (4.342634). PTCG have derived a slightly shorter period $(4.3426241 \pm 0.0000016)$, but we think that they overestimated the weight of the 
Table 2. Observing logs.

\begin{tabular}{llll}
\hline date & 1995, Oct. 21-25 & 1997, Nov. 15-21 & 1998, Dec. 2-8 \\
HJD-2 450 000 & $0011-0016$ & $0767-0774$ & $1149-1156$ \\
number of images acquired $\times$ filter & $36 \times V$ & $27 \times B+53 \times V$ & $34 \times V$ \\
range of exp. times [s] & $10-30$ & $7-30$ & $10-25$ \\
\hline
\end{tabular}

minimum published by Davidge (1987). This fact, added to the relatively short time baseline between both sets of observations, can be responsible for the small difference between both period estimates. Anyway, this little difference in the period does not affect the results.

\subsection{Spectral classification}

Niemelä \& Bassino (1994) classified the primary and secondary components of this system as $\mathrm{O} 7 \mathrm{~V}$ and $\mathrm{O} 8 \mathrm{~V}-\mathrm{III}$, respectively. More recently, based on higher S/N CCD spectra obtained at CASLEO, Niemelä \& Morrell (1999) re-classified the secondary component as B0 III; maintaining the original spectral type for the primary.

In view of the large difference between the effective temperatures derived by PTCG $(27000 \mathrm{~K}$ and $20200 \mathrm{~K}$ for the primary and secondary component, respectively) and those expected from the spectral types assigned to the components of HV 2241 we decided to review the spectral classification of this binary.

We considered ultraviolet observations downloaded from the $\mathrm{IUE}^{1}$ and $\mathrm{HST}^{2}$ archives.

We applied to these ultraviolet spectra the classification criteria described by Walborn, Nichols-Bohlin \& Panek (1985) and derived for the primary of HV 2241 a spectral type of O7 III. The spectral type, in agreement with that derived from the optical observations, is determined considering the intensities of the NV, CIV and NIV features that display strong temperature dependence, decreasing in intensity toward later spectral types. The luminosity class follows from the presence of a clear stellar wind profile in Sirv 1394-1403 $\AA$ a characteristic not observed anywhere on the main sequence. The Sirv 1394$1403 \AA$ profile is very similar in HV 2241 to that observed in HD 190864 , the O6.5 III standard star in Walborn et al. (1985). Indeed, the whole UV spectrum of HV 2241 remarkably resembles that of HD 190864 .

Figure 2 shows the ultraviolet spectrum of HV 2241 observed by the Faint Object Spectrograph on board of HST (at $\phi=0.229$ ). The spectrum of the secondary component (that we presume considerably fainter than the primary in the UV) is not observable in the analysed data.

\footnotetext{
1 Operated by the National Aeronautics and Space Administration, the European Space Agency and the Science and Engineering Research Council of the UK.

2 Operated by the Association of Universities for Research in Astronomy, Inc., under NASA contract NAS 5-26555.
}

\subsection{Light and radial velocity curve solutions}

The $V$ and $B$ CCD light curves, together with the radial velocity measurements from Niemelä \& Bassino (1994), were analysed using the last version of the WilsonDevinney (hereafter WD) program (Wilson \& Devinney 1971; Wilson 1990; Wilson 1993). We adopted a unit weight for all the photometric data, given that they all were acquired with the same telescope and detector. We point out that, although seeing conditions were rather different for different nights, this does not influence severely the obtained magnitudes in this not excessively crowded field, as indicated by the derived internal errors. For the radial velocity data from Niemelä \& Bassino (1994) we fixed a weight of 90 for the measurements near quadratures, and a weight of 10 for the data points near eclipses. The relative weight between the photometric and spectroscopic data is entered through the standard deviations (sigmas), for which we adopted a value of $0.02 \mathrm{mag}$ for the photometric data-points and $20 \mathrm{~km} \mathrm{~s}^{-1}$ for the radial velocities.

Bolometric albedos and gravity darkening coefficients were set to their theoretical values for radiative envelopes, $A=1.0$ (Rucinski 1969) and $g=1.0$ (Lucy 1976). For modelling the limb darkening we adopted a square root law (Díaz-Cordovés \& Giménez 1992), obtaining the corresponding coefficients from tables by Díaz-Cordovés et al. (1995). We also assumed synchronous rotation for both components. Given that there is a considerable spread between the different temperature calibrations for O7 III stars, ranging from $37000 \mathrm{~K}$ (Schmidt-Kaler 1982) to $39860 \mathrm{~K}$ (Vacca et al. 1996) - while Chlebowski \& Garmany (1991) give $38100 \mathrm{~K}$ - we derived alternative solutions assuming the lowest and the highest temperature scales, respectively. We used the epoch of minimum and the period calculated in the previous section. All the above mentioned parameters (i.e., albedos, gravity darkening coefficients, limb darkening coefficients, rotations and the surface temperature of the primary) were not fitted.

Then, the parameters that remained to be fitted were: the semi-major axis $a$, the systemic radial velocity $V_{\gamma}$, the orbital inclination $i$, the surface temperature of the secondary star $T_{2}$, both potentials $\Omega_{1}$ and $\Omega_{2}$ (Wilson 1979), the mass-ratio $q$ and the primary star luminosity $L_{1}$. To perform the fit, these parameters were grouped in two different sets. In the first step, the differential corrections program (DC) was used to adjust those parameters belonging to the first group, $a, q$ and $V_{\gamma}$. Thereupon, these parameters were kept constant and we improved the fit allowing the other parameter set $\left(i, T_{2}, \Omega_{1}, \Omega_{2}\right.$ and $\left.L_{1}\right)$ to 
Table 3. $V$ light photometry of HV 2241.

\begin{tabular}{|c|c|c|c|c|c|c|c|c|c|}
\hline $\begin{array}{r}\text { HJD } \\
2450000+\end{array}$ & $V$ & $\sigma_{\mathrm{i}}$ & $\begin{array}{r}F W H M \\
\prime \prime\end{array}$ & $X$ & $\begin{array}{r}\text { HJD } \\
2450000+\end{array}$ & V & $\sigma_{\mathrm{i}}$ & $\begin{array}{r}F W H M \\
\prime \prime\end{array}$ & $X$ \\
\hline 11.653 & 13.896 & 0.014 & 2.31 & 1.52 & 769.707 & 13.959 & 0.021 & 2.88 & 1.23 \\
\hline 11.670 & 13.936 & 0.010 & 2.19 & 1.46 & 769.722 & 13.949 & 0.013 & 2.50 & 1.22 \\
\hline 11.718 & 14.050 & 0.010 & 1.83 & 1.32 & 769.739 & 13.925 & 0.004 & 2.31 & 1.22 \\
\hline 11.759 & 14.136 & 0.009 & 2.51 & 1.25 & 769.770 & 13.895 & 0.018 & 1.84 & 1.22 \\
\hline 11.803 & 14.240 & 0.006 & 2.75 & 1.22 & 769.822 & 13.812 & 0.008 & 3.11 & 1.28 \\
\hline 11.824 & 14.270 & 0.005 & 2.92 & 1.22 & 770.612 & 13.490 & 0.006 & 2.51 & 1.39 \\
\hline 11.833 & 14.288 & 0.006 & 3.02 & 1.22 & 770.723 & 13.472 & 0.020 & 1.65 & 1.22 \\
\hline 11.841 & 14.299 & 0.008 & 3.94 & 1.22 & 770.773 & 13.489 & 0.006 & 2.03 & 1.23 \\
\hline 11.848 & 14.305 & 0.007 & 3.48 & 1.23 & 770.821 & 13.497 & 0.004 & 1.70 & 1.28 \\
\hline 11.854 & 14.315 & 0.006 & 3.57 & 1.23 & 770.856 & 13.495 & 0.007 & 1.52 & 1.36 \\
\hline 11.861 & 14.317 & 0.005 & 3.71 & 1.24 & 771.727 & 14.163 & 0.009 & 2.40 & 1.22 \\
\hline 11.867 & 14.320 & 0.007 & 3.48 & 1.24 & 771.750 & 14.226 & 0.008 & 2.18 & 1.22 \\
\hline 11.873 & 14.324 & 0.007 & 3.34 & 1.25 & 771.769 & 14.266 & 0.007 & 2.02 & 1.23 \\
\hline 12.646 & 13.480 & 0.007 & 3.54 & 1.54 & 771.782 & 14.276 & 0.008 & 2.05 & 1.24 \\
\hline 12.682 & 13.468 & 0.007 & 2.80 & 1.41 & 771.794 & 14.293 & 0.005 & 1.94 & 1.25 \\
\hline 12.734 & 13.461 & 0.005 & 3.58 & 1.28 & 771.807 & 14.310 & 0.007 & 2.15 & 1.27 \\
\hline 12.771 & 13.457 & 0.005 & 3.61 & 1.24 & 771.817 & 14.330 & 0.009 & 2.20 & 1.28 \\
\hline 12.815 & 13.460 & 0.009 & 4.41 & 1.22 & 771.827 & 14.330 & 0.010 & 2.38 & 1.30 \\
\hline 12.843 & 13.448 & 0.007 & 3.93 & 1.22 & 771.839 & 14.329 & 0.008 & 1.98 & 1.32 \\
\hline 12.867 & 13.453 & 0.008 & 3.97 & 1.24 & 771.847 & 14.318 & 0.006 & 1.96 & 1.34 \\
\hline 13.660 & 13.574 & 0.008 & 2.49 & 1.48 & 771.855 & 14.319 & 0.007 & 2.41 & 1.36 \\
\hline 13.708 & 13.612 & 0.007 & 2.83 & 1.33 & 771.863 & 14.309 & 0.006 & 2.81 & 1.38 \\
\hline 13.750 & 13.644 & 0.005 & 2.92 & 1.26 & 772.601 & 13.489 & 0.006 & 2.70 & 1.41 \\
\hline 13.788 & 13.678 & 0.006 & 2.69 & 1.22 & 772.668 & 13.480 & 0.004 & 2.18 & 1.26 \\
\hline 13.855 & 13.755 & 0.005 & 3.25 & 1.24 & 772.716 & 13.468 & 0.005 & 2.88 & 1.22 \\
\hline 14.643 & 13.539 & 0.006 & 3.01 & 1.53 & 772.779 & 13.458 & 0.007 & 2.51 & 1.24 \\
\hline 14.686 & 13.525 & 0.007 & 4.04 & 1.38 & 772.826 & 13.461 & 0.006 & 2.52 & 1.30 \\
\hline 14.734 & 13.518 & 0.005 & 3.93 & 1.27 & 773.581 & 13.574 & 0.006 & 1.93 & 1.47 \\
\hline 14.774 & 13.514 & 0.009 & 4.90 & 1.23 & 773.639 & 13.601 & 0.007 & 1.86 & 1.31 \\
\hline 14.856 & 13.495 & 0.006 & 4.39 & 1.24 & 773.725 & 13.670 & 0.006 & 2.34 & 1.22 \\
\hline 15.622 & 13.573 & 0.006 & 3.02 & 1.62 & 773.765 & 13.713 & 0.009 & 2.25 & 1.23 \\
\hline 15.686 & 13.592 & 0.010 & 2.82 & 1.37 & 773.799 & 13.745 & 0.006 & 2.99 & 1.26 \\
\hline 15.734 & 13.608 & 0.007 & 3.10 & 1.27 & 773.838 & 13.785 & 0.011 & 2.12 & 1.33 \\
\hline 15.783 & 13.637 & 0.005 & 3.04 & 1.22 & 1149.782 & 14.051 & 0.008 & 2.32 & 1.28 \\
\hline 15.829 & 13.670 & 0.006 & 3.81 & 1.22 & 1149.823 & 13.938 & 0.006 & 1.89 & 1.37 \\
\hline 15.860 & 13.701 & 0.005 & 3.21 & 1.25 & 1150.680 & 13.459 & 0.017 & 1.59 & 1.22 \\
\hline 767.625 & 14.072 & 0.007 & 2.11 & 1.38 & 1150.834 & 13.452 & 0.018 & 1.59 & 1.41 \\
\hline 767.687 & 13.932 & 0.006 & 2.09 & 1.26 & 1151.565 & 13.706 & 0.006 & 1.67 & 1.41 \\
\hline 767.758 & 13.795 & 0.005 & 2.55 & 1.22 & 1151.626 & 13.779 & 0.005 & 1.80 & 1.27 \\
\hline 767.802 & 13.724 & 0.004 & 2.61 & 1.25 & 1151.675 & 13.847 & 0.004 & 1.60 & 1.22 \\
\hline 767.844 & 13.672 & 0.004 & 2.54 & 1.31 & 1151.727 & 13.903 & 0.004 & 1.73 & 1.23 \\
\hline 768.620 & 13.462 & 0.011 & 2.70 & 1.38 & 1151.744 & 13.928 & 0.006 & 1.70 & 1.24 \\
\hline 768.673 & 13.461 & 0.008 & 2.78 & 1.27 & 1151.763 & 13.932 & 0.005 & 1.71 & 1.26 \\
\hline 768.742 & 13.465 & 0.007 & 1.92 & 1.22 & 1151.777 & 13.948 & 0.006 & 1.81 & 1.28 \\
\hline 768.794 & 13.476 & 0.006 & 2.31 & 1.24 & 1151.794 & 13.957 & 0.007 & 2.04 & 1.31 \\
\hline 768.836 & 13.484 & 0.007 & 2.02 & 1.30 & 1151.813 & 13.966 & 0.006 & 2.15 & 1.36 \\
\hline 768.866 & 13.469 & 0.013 & 1.89 & 1.37 & 1151.830 & 13.965 & 0.005 & 1.96 & 1.41 \\
\hline 769.594 & 13.927 & 0.005 & 3.11 & 1.46 & 1151.850 & 13.964 & 0.005 & 1.85 & 1.48 \\
\hline 769.619 & 13.949 & 0.006 & 2.28 & 1.38 & 1151.861 & 13.947 & 0.008 & 1.91 & 1.52 \\
\hline 769.633 & 13.955 & 0.006 & 2.44 & 1.34 & 1152.670 & 13.488 & 0.006 & 1.84 & 1.22 \\
\hline 769.644 & 13.966 & 0.006 & 2.43 & 1.32 & 1152.722 & 13.479 & 0.006 & 1.84 & 1.22 \\
\hline 769.654 & 13.974 & 0.009 & 2.79 & 1.30 & 1152.756 & 13.484 & 0.005 & 1.50 & 1.26 \\
\hline 769.663 & 13.980 & 0.006 & 2.14 & 1.28 & 1152.807 & 13.479 & 0.005 & 1.58 & 1.35 \\
\hline 769.674 & 13.972 & 0.005 & 2.36 & 1.26 & 1152.837 & 13.470 & 0.007 & 1.78 & 1.44 \\
\hline 769.686 & 13.954 & 0.024 & 2.54 & 1.25 & 1153.702 & 13.806 & 0.004 & 1.40 & 1.22 \\
\hline
\end{tabular}


Table 3. continued.

\begin{tabular}{rrrrrrrrrr}
\hline $\begin{array}{r}\text { HJD } \\
2450000+\end{array}$ & $V$ & $\sigma_{\mathrm{i}}$ & $\begin{array}{r}F W H M \\
\prime \prime\end{array}$ & $X$ & $\begin{array}{r}\text { HJD } \\
2450000+\end{array}$ & $V$ & $\sigma_{\mathrm{i}}$ & $\begin{array}{r}F W H M \\
\prime \prime\end{array}$ & $X$ \\
\hline 1153.747 & 13.871 & 0.006 & 1.47 & 1.25 & 1154.852 & 13.476 & 0.005 & 2.18 & 1.52 \\
1153.780 & 13.938 & 0.006 & 1.95 & 1.30 & 1155.680 & 13.553 & 0.007 & 1.61 & 1.22 \\
1153.839 & 14.058 & 0.005 & 2.85 & 1.46 & 1155.720 & 13.565 & 0.006 & 1.79 & 1.23 \\
1154.712 & 13.507 & 0.005 & 2.01 & 1.22 & 1155.754 & 13.576 & 0.008 & 1.72 & 1.26 \\
1154.749 & 13.499 & 0.005 & 1.73 & 1.25 & 1155.785 & 13.601 & 0.008 & 1.64 & 1.32 \\
1154.824 & 13.471 & 0.007 & 2.26 & 1.41 & 1155.835 & 13.645 & 0.005 & 2.14 & 1.46 \\
\hline
\end{tabular}

Table 4. B light photometry of HV 2241.

\begin{tabular}{rrrrrrrrrr}
\hline $\begin{array}{r}\text { HJD } \\
2450000+\end{array}$ & $B$ & $\sigma_{\mathrm{i}}$ & $\begin{array}{r}F W H M \\
\prime \prime\end{array}$ & $X$ & $\begin{array}{r}\text { HJD } \\
2450000+\end{array}$ & $B$ & $\sigma_{\mathrm{i}}$ & $\begin{array}{r}F W M \\
\prime \prime\end{array}$ & $X$ \\
\hline 769.595 & 13.868 & 0.005 & 3.45 & 1.46 & 771.783 & 14.260 & 0.007 & 2.32 & 1.24 \\
769.621 & 13.888 & 0.008 & 2.36 & 1.38 & 771.796 & 14.283 & 0.006 & 2.66 & 1.25 \\
769.635 & 13.900 & 0.006 & 2.23 & 1.34 & 771.809 & 14.302 & 0.009 & 2.42 & 1.27 \\
769.645 & 13.917 & 0.006 & 2.24 & 1.32 & 771.819 & 14.315 & 0.006 & 2.35 & 1.28 \\
769.664 & 13.916 & 0.006 & 2.24 & 1.28 & 771.830 & 14.316 & 0.011 & 2.14 & 1.31 \\
769.676 & 13.914 & 0.009 & 2.17 & 1.26 & 771.840 & 14.317 & 0.010 & 2.63 & 1.33 \\
769.692 & 13.888 & 0.006 & 2.68 & 1.24 & 771.849 & 14.311 & 0.006 & 2.30 & 1.35 \\
769.709 & 13.888 & 0.005 & 3.25 & 1.23 & 771.856 & 14.300 & 0.004 & 2.50 & 1.37 \\
769.741 & 13.863 & 0.013 & 2.48 & 1.22 & 771.864 & 14.305 & 0.005 & 2.79 & 1.39 \\
769.776 & 13.824 & 0.004 & 2.51 & 1.23 & 772.600 & 13.450 & 0.006 & 2.42 & 1.41 \\
769.825 & 13.751 & 0.018 & 2.50 & 1.29 & 772.715 & 13.429 & 0.007 & 2.79 & 1.22 \\
771.728 & 14.160 & 0.007 & 2.50 & 1.22 & 772.776 & 13.418 & 0.006 & 2.79 & 1.23 \\
771.752 & 14.201 & 0.006 & 2.10 & 1.22 & 772.825 & 13.408 & 0.006 & 2.94 & 1.30 \\
771.770 & 14.247 & 0.006 & 2.20 & 1.23 & & & & & \\
\hline
\end{tabular}

vary. Then we re-adjusted the first parameter set keeping constant the second, and repeated these two steps iteratively. Even starting with different sets of initial values, the solution rapidly converged towards a semi-detached configuration, with the coolest and less massive star filling its Roche-lobe. Consequently, we used the operation mode 5 of the DC program in successive iterations. In that mode, the surface potential of the secondary star (i.e., the B0 III component) is kept equal to the critical Roche-lobe surface potential and is not fitted. A better approximation was reached by including the mass-ratio in the second parameter set.

Visual examination of the light curve allows one to verify the presence of the O'Connell effect, i.e., a slight brightness difference between both maxima (Davidge \& Milone 1984). This feature was also noted in the previous studies by Davidge (1987) and PTCG. Since there is not a definitive explanation about the O'Connell effect in hot stars, we simply modelled it by adding a "hot-spot" on one side of one of the stars. This seems an arbitrary procedure, but it is only a "cosmetic" patch, that does not significantly influence the solution.

Finally, when the obtained parameters oscillated around the final values, we allowed all the parameters to be adjusted simultaneously. Figures 3 and 4 show the modelled light and radial velocity curves, respectively, plotted along with the observations, and their corresponding residuals $(\mathrm{O}-\mathrm{C})$. The perturbed shape of the velocity curve during eclipses is due to the so called Rossiter or Schlesinger effect (Rossiter 1924; Schlesinger 1910).

We also performed another fit including also the data from PTCG, that is, photometric measurements in the Strömgren $u, v, b$ and $y$ bands and Cousins $I$ band, finding no meaningful differences in the results. Our finally adopted model parameters and the resulting star dimensions are displayed in Tables 5 and 6 .

\section{Discussion}

Although our solution agrees with that of PTCG concerning the masses, radii and orbital elements, our surface temperatures are very much higher than those obtained by them. We recall that we do not derive a new temperature for the primary component, but simply adopted the temperature corresponding to the spectral type of the primary star from other works (Schmidt-Kaler 1982; Chlebowsky \& Garmany 1991; Vacca et al. 1996). The secondary star's temperature is then reflected by the relative depth of the eclipses. For the different temperature scales adopted for the primary, we obtain $T_{2}$ ranging from $28300 \mathrm{~K}$ to $30700 \mathrm{~K}$. This is in agreement with its spectral type according to Schmidt-Kaler $(29000 \mathrm{~K}$ for a B0 III star), while Vacca et al. give $31540 \mathrm{~K}$. On the other hand, PTCG derived the temperatures from their own 


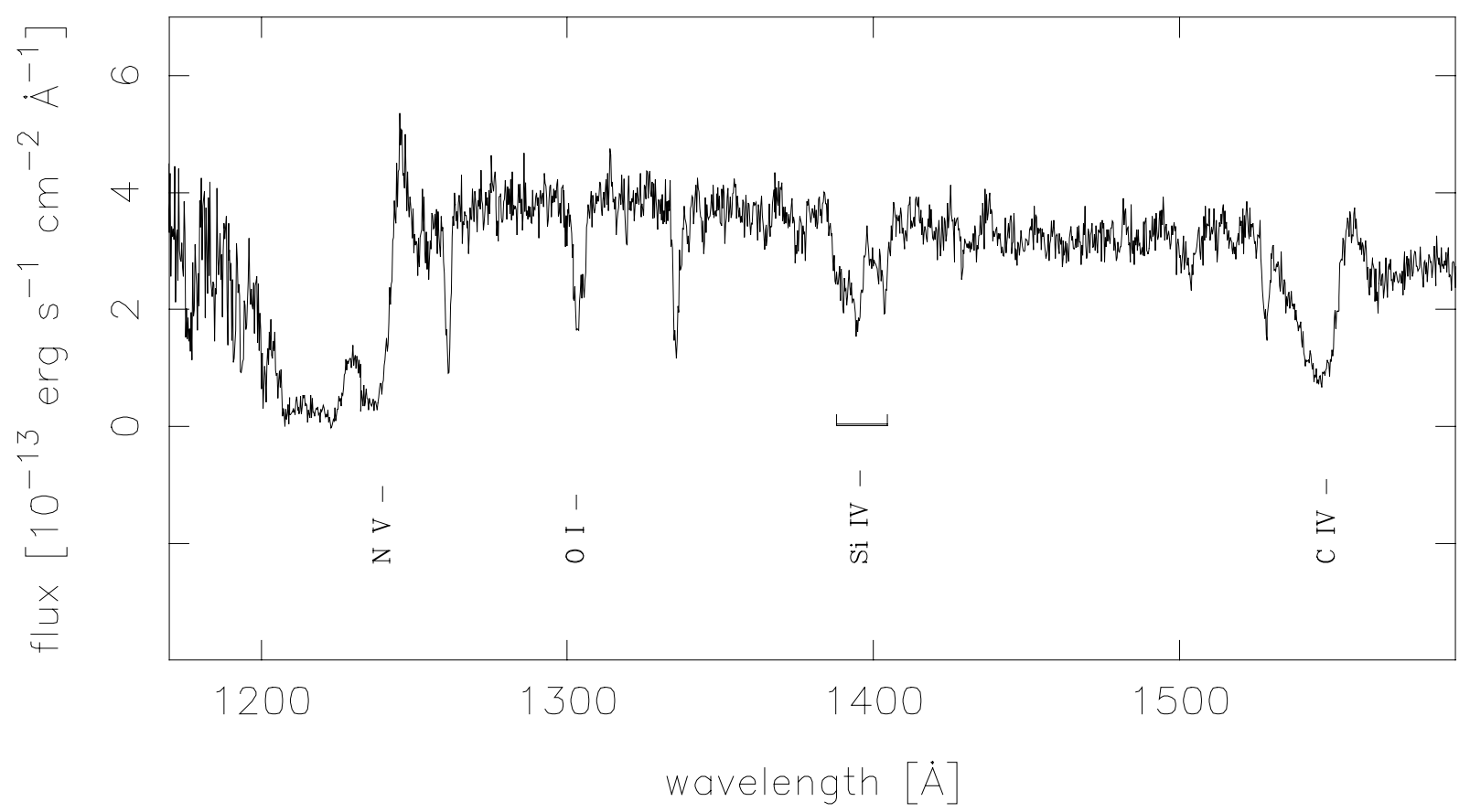

Fig. 2. HST/FOS spectrum of HV 2241 used for spectral classification. Note the wind profile in Sirv $1394-1403$ characteristic of luminosity class III.

Table 5. Model parameters.

\begin{tabular}{ll}
\hline$a$ & $42.5 \pm 0.5 R_{\odot}$ \\
$V_{\gamma}$ & $307.9 \pm 3 \mathrm{~km} \mathrm{~s}^{-1}$ \\
$i$ & $84 \pm 1^{\circ}$ \\
$q\left(M_{2} / M_{1}\right)$ & $0.51 \pm 0.04$ \\
$T_{1}$ & $37000-39860 \mathrm{~K}$ (adopted) \\
$\Omega_{1}$ & $3.417 \pm 0.08$ \\
$g_{1}$ & 1.00 (adopted) \\
$A_{1}$ & 1.00 (adopted) \\
$T_{2}$ & $28300-30700 \mathrm{~K}^{\mathrm{a}}$ \\
$\Omega_{2}$ & $2.892 \pm 0.08$ \\
$g_{2}$ & 1.00 (adopted) \\
$A_{2}$ & 1.00 (adopted) \\
\hline
\end{tabular}

\begin{tabular}{ll}
\hline & Hot spot (in star 1): \\
colatitude & $90^{\circ}$ \\
longitude & $270^{\circ}$ \\
angular radius & $57.3^{\circ}$ \\
temp. factor & 1.035 \\
\hline
\end{tabular}

a Values of $T_{2}$ resulting from the above quoted values adopted for $T_{1}$, respectively.

multi-band photometry and IUE spectrophotometry, so that the strong departures between both estimates do not result from "different solutions" to the system but from alternative methods to derive them.

From our $B V$ photometry we obtain $(B-V)=$ $-0.039 \pm 0.015$, while, according to the spectral types, the intrinsic colour index for this system should be $(B-$ $V)_{0} \approx-0.31$. Consequently, we estimate a colour excess
Table 6. Star dimensions.

\begin{tabular}{ll}
\hline$M_{1}$ & $36.23 \pm 0.7 M_{\odot}$ \\
$R_{1}$ & $14.94 \pm 0.4 R_{\odot}$ \\
$M_{\text {bol } 1}$ & $-9.15--9.47^{\mathrm{a}}$ \\
$\log g_{1}[\mathrm{cgs}]$ & $3.65 \pm 0.03$ \\
$M_{2}$ & $18.41 \pm 0.7 M_{\odot}$ \\
$R_{2}$ & $13.67 \pm 0.4 R_{\odot}$ \\
$M_{\text {bol } 2}$ & $-7.79--8.15^{\mathrm{a}}$ \\
$\log g_{2}[\mathrm{cgs}]$ & $3.43 \pm 0.01$
\end{tabular}

a The range in the derived bolometric magnitudes corresponds to the range in the values adopted for $T_{1}$.

$E(B-V)=0.27$, a value significantly higher than that adopted by PTCG $(0.174 \pm 0.020)$.

When comparing both results, we should consider some facts:

- In our approach, we assign an intrinsic colour to the system, according to the spectral types of its components. The validity of this procedure depends on the reliability of the colour vs. spectral type calibrations. We emphasize that, although the temperature corresponding to an O7 III star ranges through a wide interval according to the different calibrations, the $(B-$ $V$ ) colour is not temperature sensitive for hot stars, therefore the assumed intrinsic colour should be quite realistic;

- PTCG determine the colour excess using the empirical $(b-y)_{0}$ vs. $c_{0}$ relation and a $E\left(c_{1}\right)=0.2 E(b-y)$ reddening ratio, but this only works provided that a universal extinction law holds. There are regions in the 


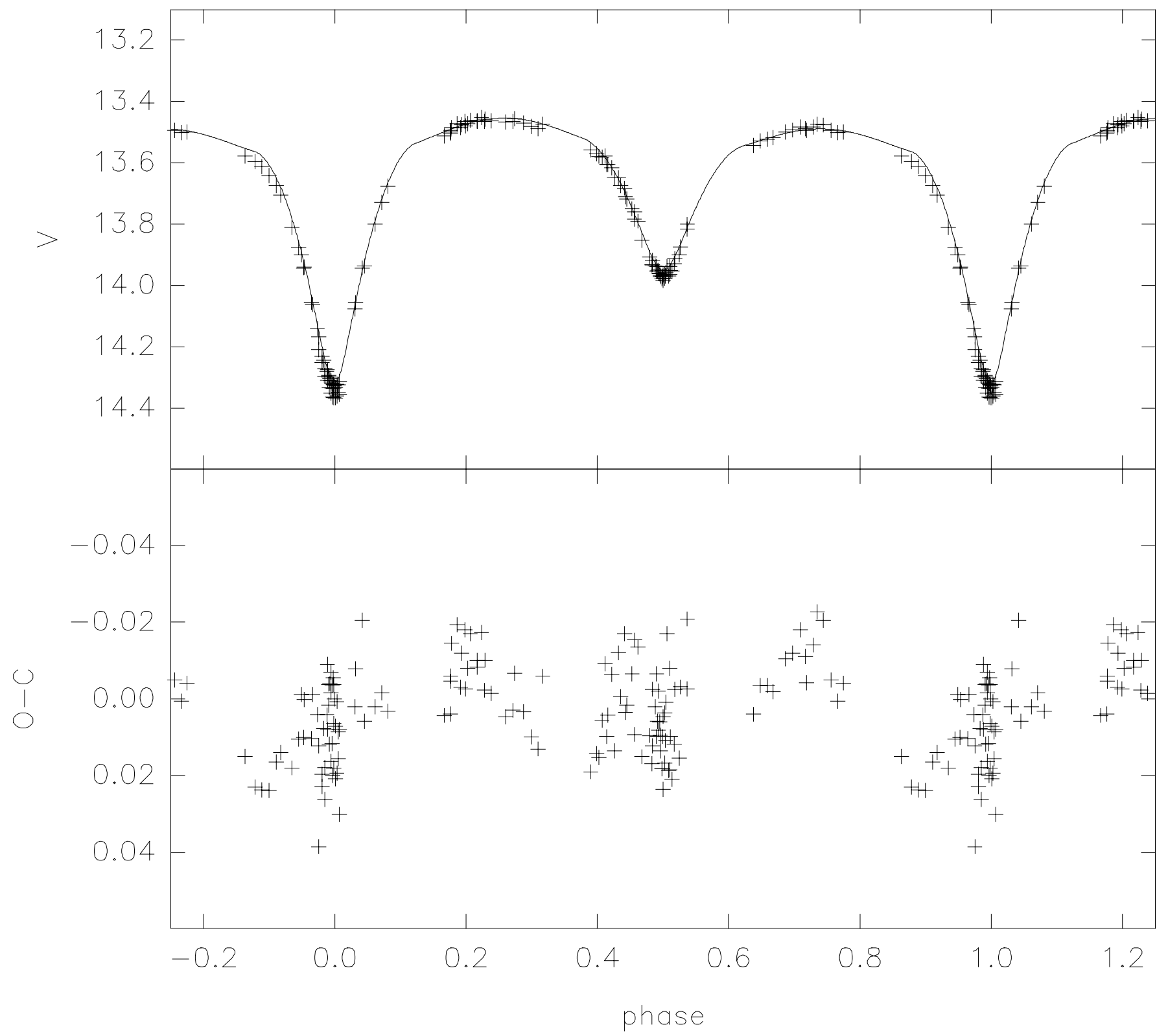

Fig. 3. Top: Observed and modelled light curve for HV 2241. Bottom: (O-C) residuals for the light curve.

galaxy that exhibit notable departures in their interstellar extinction laws, and presumably the same case occurs in the LMC;

- Besides these points, the difference between both photometries is small, but larger than the errors. PTCG derive $(b-y)=0.004 \pm 0.008$ and $m_{1}=0.047 \pm 0.013$ (for $\phi=0.832)$. Converting these indexes to $(B-V)$ by means of the transformation relations for early type stars given by Turner (1990), we obtain $(B-V)=$ -0.077 , while our value is $(B-V)=-0.039$. Since these colour indexes are not strongly affected by line blanketing, we do not expect that metallicity effects can very much deviate these transformations. On the other hand, PTCG derived $V=13.51$ (for $\phi=0.832$ ), in excellent agreement with our photometry, that (according to our light curve solution), yields $V=13.52$ for that phase.
The reason for the disagreement between both reddening estimates can be a combination of all these factors. Given that the IUE observations do not reach the maximum of the flux distribution of this early system, a slight difference in the reddening would strongly affect the derived temperature, thus explaining the large discrepancy between the spectrophotometric temperatures and those expected from spectral types.

Masses for single stars can be estimated indirectly using stellar atmosphere models, either analysing line profiles or measuring the wind terminal velocity. It is known that systematic differences exist, for the earliest type stars, between these "spectroscopic" masses and those predicted by numerical evolutive models, in the sense that spectroscopic masses are lower than the evolutive ones (see, for example, Groenewegen et al. 1989; Herrero et al. 1992). This so called "mass discrepancy" can be reduced (but 


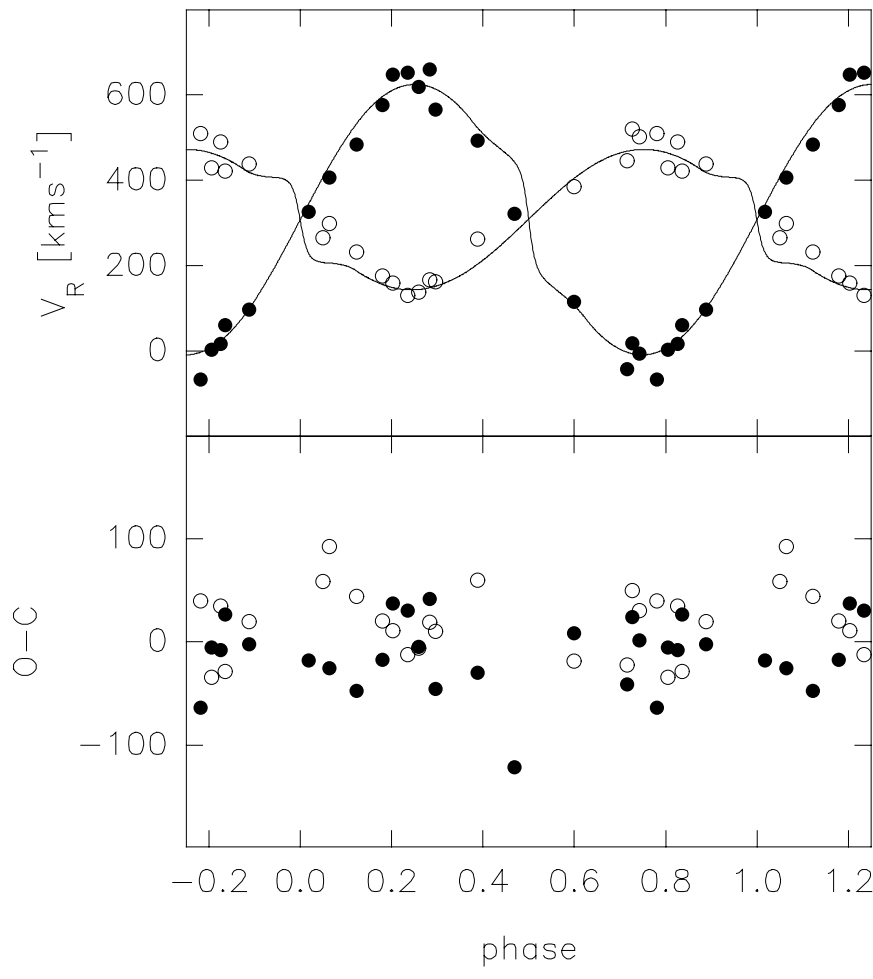

Fig. 4. Top: observed and modelled radial velocity curve for HV 2241. Bottom: (O-C) residuals for the radial velocities. Hollow circles correspond to the primary component and filled ones stand for the secondary.

no eliminated) by means of more detailed model atmospheres (Lanz et al. 1996; Herrero et al. 2000). Regarding the evolutionary computations for single stars, changes in the mass loss rate, overshooting parameter, and stellar rotation effects can dramatically alter the theoretical evolutive tracks for the very massive objects (see, for example, Maeder \& Meynet 2000; Meynet \& Maeder 2000).

The only direct method to obtain stellar masses is by means of the simultaneous analysis of light and radial velocity curves of double lined eclipsing binaries, but the more massive systems evolve via Roche lobe overflow and mass exchange, a fact that introduces additional complications for this approach.

From our solution, we presume that HV 2241 experienced case A mass transfer and that mass inversion occurred. It is expected that the mass gainer (i.e. the O7 III component in our case) should be rejuvenated but still similar to a normal star concerning its mass, temperature and radius (Vanbeveren et al. 1998). From the evolutive tracks computed by Schaerer et al. (1993), we find that a star with an initial mass of $40 M_{\odot}$ will have, after 3.8 Myr, a mass of $\sim 39 M_{\odot}$, a radius of $\sim 15 R_{\odot}$, and a surface temperature of $\sim 37000 \mathrm{~K}$, values that are not far from those derived here. However, the comparison with the mass and radius found for the O8 component of HV 2543 (Ostrov et al. 2000) is puzzling: both stars have similar radii, $\sim 14.9 R_{\odot}$ and $\sim 15.5 R_{\odot}$, close spectral types, O7 III and O8 III (and hence similar surface temperatures), but rather different masses, namely

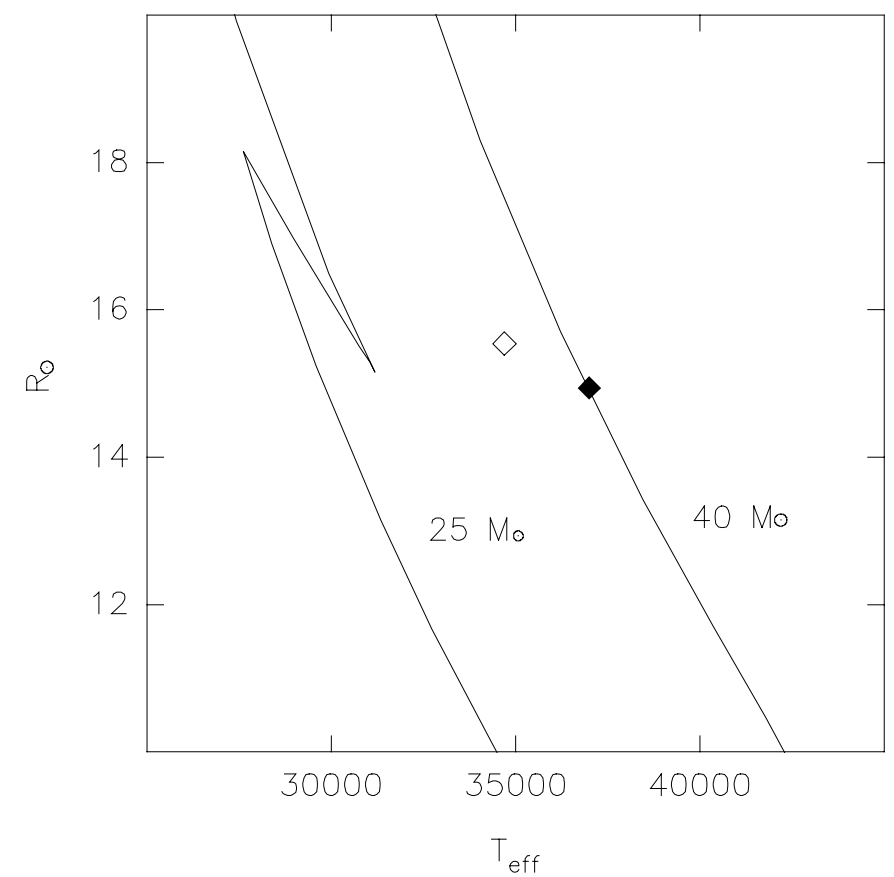

Fig. 5. The radii and effective temperatures of the mass gainer components of HV 2543 (white diamond) and HV 2241 (black diamond), plotted together with the models of Schaerer et al. (1993).

$\sim 36.2 M_{\odot}$ and $\sim 25.6 M_{\odot}$, respectively. In Fig. 5 we plotted the radii and effective temperatures of the primaries of HV 2241 and HV 2543, together with the tracks from Schaerer et al. (1993). As we can see, the mass we found for the O7 III star is only $\sim 3 M_{\odot}$ lower that the value derived from the evolutionary track, while the O8 III component of HV 2543 is excessively hot compared with models for its mass. We presume that the O8 III star is in a more advanced evolutionary stage, that might suggest that the discrepancies with the models grow as evolution proceeds. Nevertheless, it is also possible that some aspects of the binary evolution theory still need to be clarified.

With regard to the distance modulus, using the bolometric corrections by Massey \& Hunter (1998), and adopting $R=3.1$ from Koornneef $(1982)$, we obtain $(m-M)_{0}=$ 18.65 or 18.80 according to which temperature scale is adopted. Of course, besides the inherent uncertainties of dimensions and temperatures, this value only holds if $R=3.1$ is a good guess.

\section{Conclusions}

From the analysis of our $B$ and $V$ photometry as well as published spectroscopic observations of the Magellanic eclipsing binary HV 2241, we confirmed that this system is semidetached, with the cooler and less massive component filling its Roche lobe. For this system, we derived the following physical parameters: $M_{1}=36.2 M_{\odot}$, $M_{2}=18.4 M_{\odot}, R_{1}=14.9 R_{\odot}$ and $R_{2}=13.7 R_{\odot}$, with an inclination of $84^{\circ}$. 
We reviewed the spectral classification using public IUE and HST spectroscopic data, finding that the most reliable spectral type for the primary star is O7 III.

We estimated a value for the colour excess for this star - $E(B-V)=0.27$ - that is significantly larger than that previously published, and can be the origin of the large differences in temperature estimations.

Acknowledgements. The authors acknowledge use at CASLEO of the CCD and data acquisition system supported under U.S. National Science Foundation grant AST-90-15827 to R. M. Rich. The focal reducer in use at CASLEO was kindly provided by Dr. M. Shara. We are indebted to the staff of CASLEO for valuable help during the observing runs. Based on observations made with the NASA/ESA Hubble Space Telescope, obtained from the data archive at the Space Telescope Science Institute. STScI is operated by the Association of Universities for Research in Astronomy, Inc. under NASA contract NAS 5-26555. The authors want to thank Dr. Virpi Niemelä for suggesting the study of HV 2241 and other Harvard variables. We wish to thank the referee, A. F. J. Moffat, for his suggestions and careful reading of the manuscript, and especially for his patience and dedication in order to improve our English.

\section{References}

Antokhina, E. A., Moffat, A. F. J., Antokhin, I. I., Bertrand, J.-F., \& Lamontagne, R. 2000, ApJ, 529, 463

Burkholder, V., Massey, P., \& Morrell, N. 1997, ApJ, 490, 328

Chlebowski, T., \& Garmany, C. D. 1991, ApJ, 368, 241

Davidge, T. J. 1987, AJ, 94, 1169

Davidge, T. J., \& Milone, E. F. 1984, ApJS, 55, 571

Díaz-Cordovés, J., \& Giménez, A., A\&A, 259, 227

Díaz-Cordovés, J., Claret, A., \& Giménez, A., A\&AS, 110, 329

Gaposhkin, S. 1970, Smithsonian Ast. Obs. Spec. Rep., 310

Groenewegen, M. A. T., Lamers, H. J. G. L. M., \& Pauldrach, A. W. A. 1989, A\&A, 221, 78

Herrero, A., Kudritzki, R. P., Vilchez, J. M., et al. 1992, A\&A, 261, 209

Herrero, A., Puls, J., \& Villamariz, M. R. 2000, A\&A, 354, 193

Koornneef, J. 1982, A\&A, 107, 247

Landolt, A. U. 1992, AJ, 104, 340
Lanz, T., de Koter, A., Hubeny, I., \& Heap, S. R. 1996, ApJ, 465,359

Lucy, L. B. 1976, ApJ, 205, 208

Massey, P., \& Hunter, D. A. 1998, ApJ, 493, 180

Maeder, A., \& Meynet, G. 2000, A\&A, 361, 159

Meynet, G., \& Maeder, A. 2000, A\&A, 361, 101

Minniti, D., Clariá, J. J., \& Gómez, M. N. 1989, Ap\&SS, 158, 9

Rossiter, R. A. 1924, ApJ, 60, 15

Schlesinger, F. 1910, Pub. of the Allegheny Obs., 1, 123

Niemelä, V. S., \& Bassino, L. P. 1994, ApJ, 437, 332

Niemelä, V. S., \& Morrell, N. I. 1999, in New Views of the Magellanic Clouds, ed. Y.-H. Chu, N. Suntzeff, J. E. Hesser, \& D. Bohlender, IAU Symp., 190, 181

Ostrov, P. G. 2001, MNRAS, 321, L25

Ostrov, P. G., Lapasset, E., \& Morrell, N. I. 2000, A\&A, 356, 935

Payne-Gaposchkin, C. H. 1971, Smithsonian Contr. to Astrophysics, No. 13

Pritchard, J. D., Tobin, W., Clark, M., \& Guinan, E. F. 1998, MNRAS, 297, 278 [PTCG]

Rucinski, S. M. 1969, Acta Astron., 19, 245

Russell, H. N. 1956, Vistas Astron., 2, 1177

Schaerer, D., Meynet, G., Maeder, A., \& Schaller, G. 1993, A\&AS, 98, 523

Schmidt-Kaler, Th. 1982, in Landolt-Börnstein, New Series, ed. K. Shaifers, \& H. H. Voigt, Group VI, vol. 2/b

Schweickhardt, J., Schmutz, W., Stahl, O., Szeifer, Th., \& Wolf, B. 1999, A\&A, 347, L27

Stetson, P. B. 1987, PASP, 99, 191

Stetson, P. B. 1991, in 3rd ESO/ST-ECF Data Analysis

Turner, D. G. 1990, PASP, 102, 1331

Vacca, W. D., Garmany, C. D., \& Shull, J. M. 1996, ApJ, 460, 914

Vanbeveren, D., De Loore, C., \& Van Rensbergen, W. 1998, A\&AR, 9, 63

Walborn, N. R., Nichols-Bohlin, J., \& Panek, R. J. 1985, International Ultraviolet Explorer Atlas of O-Type Spectra from 1200 to $1900 \AA$ (NASA RP-1155)

Wellstein, S., Langer, N., \& Braun, H. 2001, A\&A, 369, 939

Wilson, R. E. 1979, ApJ, 234, 1054

Wilson, R. E. 1990, ApJ, 356, 613

Wilson, R. E., in New frontiers in Binary Star Research, ed. K. C. Leung, \& I. S. Nha, ASP Conf. Ser., 38, 91

Wilson, R. E., \& Devinney, E. J. 1971, ApJ, 166, 605 